\title{
Selecting the Right Tumors for Genomic Testing
}

\author{
David M. Euhus, MD
}

Department of Surgery, Johns Hopkins Hospital, Baltimore, MD

The adult female breast comprises 10-15 independent ductal systems. ${ }^{1}$ Ideally, separate cancers arising in the same duct system would be classified as multifocal while those arising in different duct systems would be multicentric. Theoretically, multifocal cancers would be quite similar to each other, while multicentric cancers could be quite different. Unfortunately, there is currently no reliable way to determine the duct system of origin for any given breast cancer. Tumors arising in the same quadrant are commonly classified as multifocal, while those arising in different quadrants are classified as multicentric. There is some validity to this approach, as mutation analysis finds that the most disparate tumors are often further apart. ${ }^{2}$ Nevertheless, the distinction is artificial, so the term multifocal/multicentric (MF/MC) is more accurate.

Serial sectioning of mastectomy specimens suggests that more than $50 \%$ of breast cancers are $\mathrm{MF} / \mathrm{MC}^{3-5}$ The success of breast conservation attests to the fact that it is unnecessary to identify and specifically deal with each focus. Increasingly, MF/MC breast cancer is identified by clinical breast imaging. Modern series suggest that about $20 \%$ of breast cancers are MF/MC at diagnosis, ${ }^{6,7}$ with higher rates for luminal $\mathrm{B}$ and human epidermal growth factor receptor (HER)2(+) breast cancer. ${ }^{8}$ The individual tumor foci are morphologically similar and share a biomarker profile in upwards of $90 \%$ of MF/MC cases. ${ }^{6}$ Because of this, it is often reasonable to stage and make treatment decisions based on the largest focus, as is recommended by the Breast College of American Pathologists (CAP) protocol. ${ }^{9}$ However, when the tumor foci differ in grade and histology, the biologically more aggressive focus

(C) Society of Surgical Oncology 2018

First Received: 23 October 2018;

Published Online: 12 November 2018

D. M. Euhus, MD

e-mail: deuhus1@jhmi.edu is more frequently associated with lymph node metastases, regardless of size, and should be the driver of treatment decisions.

In the modern era, size and grade have largely been supplanted by biological markers such as estrogen receptor (ER), progesterone receptor, and HER2/neu (HER2). For the ER(+)/HER2(-) subset, genomic profiling provides additional prognostic information and identifies women unlikely to benefit from chemotherapy. In this issue of Annals of Surgical Oncology, Grabenstetter et al. ${ }^{10}$ ask whether morphologically similar, ER(+)/HER2(-) MF/ MC tumor foci share genomic profiles provided by the OncoTypeDX test. This retrospective series included women with lymph-node-negative $\mathrm{ER}(+) / \mathrm{HER} 2(-) \mathrm{MF} /$ MC breast cancer $\geq 0.5 \mathrm{~cm}$ who had an OncoTypeDX result for more than one tumor focus. This latter criterion appears to have enriched the series for MF/MC tumors with morphologically dissimilar foci, but these were excluded, leaving 53 patients with morphologically similar tumors for analysis. The OncoTypeDX tests assigned all tumor foci to the same risk category in $87 \%$ of patients. The authors conclude that testing one tumor focus in MF/MC cases with similar morphology is enough. This conclusion is well supported by the data.

The OncoTypeDX test measures the expression of 16 genes, mostly related to proliferation and sex hormone signaling, compared with the expression of 5 reference genes. ${ }^{11}$ The output is a recurrence score that ranges from 0 to 100. A low risk score predicts excellent prognosis with endocrine therapy only, while a high risk score predicts benefit from chemotherapy. The recently published TailoRX trial found that only $14 \%$ of early-stage patients with ER(+)/HER2(-) tumors benefit from chemotherapy. ${ }^{12}$ The value of the test is to identify patients who do not need to receive chemotherapy. National Comprehensive Cancer Network (NCCN) guidelines state "strongly consider 21-gene RT-PCR assay" for any lymph-node-negative $\mathrm{ER}(+) / \mathrm{HER} 2(-)$ invasive breast cancer $>0.5 \mathrm{~cm} .{ }^{13}$ It can 
be conservatively estimated that, in the USA, there would be 100,000 women who meet these criteria each year, adding more than US $\$ 300,000,000$ to the cost of caring for breast cancer. However, avoidance of chemotherapy represents a significant cost saving, and a cost-benefit model concluded that the test is reasonably cost effective. ${ }^{14}$ Nevertheless, Grabenstetter et al. are right in trying to reduce the number of expensive genomic tests that are obtained, and it is reasonable to try and reduce this number further.

Two studies have reported that $<5 \%$ of stage $1-2$ breast cancers that are grade 1 and progesterone receptor positive have a high risk recurrence score. ${ }^{15,16}$ Theoretically, these tumors do not need genomic testing. Wolff et al. developed a nomogram for predicting OncoTypeDX score based on ER, progesterone receptor, proliferation (Ki67), and grade. ${ }^{17}$ Their nomogram predicts the OncoTypeDX risk classification with $>95 \%$ confidence in $>55 \%$ of tumors. This provides another way to incorporate pathologic information into clinical decision-making so that genomic tests can be ordered when they are most likely to add new information.

Some might argue that the $87 \%$ concordance reported by Grabenstetter et al. for $\mathrm{MF} / \mathrm{MC}$ tumors is not high enough and could leave $13 \%$ of tumors misclassified. Further analysis of their discordant cases showed that foci with higher risk scores were associated with biopsy site changes and foci associated with lower risk scores were associated with regions of extensive ductal carcinoma in situ. This highlights the importance of careful selection of tissue blocks for genomic testing.

Genomic testing contributes substantially to individualized breast cancer treatment consistent with modern precision medicine goals. The test is expensive, and overuse should be avoided. For women with multiple tumor foci that are morphologically similar, the largest focus can be selected for testing.

\section{REFERENCES}

1. Going JJ, Moffat DF. Escaping from Flatland: clinical and biological aspects of human mammary duct anatomy in three dimensions. J Pathol. 2004;203(1):538-544.
2. Desmedt C, Fumagalli D, Pietri E, et al. Uncovering the genomic heterogeneity of multifocal breast cancer. $J$ Pathol. 2015;236(4):457-466.

3. Gallager HS, Martin JE. The study of mammary carcinoma by mammography and whole organ sectioning. Early observations. Cancer. 1969;23(4):855-873.

4. Holland R, Veling SH, Mravunac M, Hendriks JH. Histologic multifocality of Tis, T1-2 breast carcinomas. Implications for clinical trials of breast-conserving surgery. Cancer. 1985;56(5):979-990.

5. Vaidya JS, Vyas JJ, Chinoy RF, Merchant N, Sharma OP, Mittra I. Multicentricity of breast cancer: whole-organ analysis and clinical implications. Br J Cancer. 1996;74(5):820-824.

6. Singh K, Wang Y, Marketkar S, Kalife ET, Steinhoff MM. Comparison of estrogen receptor, progesterone receptor and HER2 results in concurrent ipsilateral samples with invasive breast carcinoma: a retrospective study of 246 biopsies from 119 patients. Hum Pathol. 2017;65:123-132.

7. Boros M, Podoleanu C, Georgescu R, Moldovan C, Molnar C, Stolnicu S. Multifocal/multicentric breast carcinomas showing intertumoural heterogeneity: a comparison of histological tumour type and Nottingham histological grade of primary tumour and lymph node metastasis. Pol J Pathol. 2015;66(2):125-132.

8. Ha R, Jin B, Mango V, et al. Breast cancer molecular subtype as a predictor of the utility of preoperative MRI. AJR Am J Roentgenol. 2015;204(6):1354-1360.

9. Lester SC, Bose S, Chen YY, et al. Protocol for the examination of specimens from patients with invasive carcinoma of the breast. Arch Pathol Lab Med. 2009;133(10):1515-1538.

10. Grabenstetter A, Brogi E, Chou JF, et al. Multifocal/multicentric ipsilateral invasive breast carcinomas with similar histology: is multigene testing of all individual foci necessary? Ann Surg Oncol. 2018. https://doi.org/10.1245/s10434-018-6866-y.

11. Sparano JA, Paik S. Development of the 21-gene assay and its application in clinical practice and clinical trials. J Clin Oncol. 2008;26(5):721-728.

12. Sparano JA, Gray RJ, Makower DF, et al. Adjuvant chemotherapy guided by a 21-gene expression assay in breast cancer. $N$ Engl J Med. 2018;379(2):111-121.

13. Breast Cancer. NCCN Clinical Practice Guidelines in Oncology (NCCN Guidelines) 2018; 2.2018. Accessed October 20, 2018.

14. Chandler Y, Schechter CB, Jayasekera J, et al. Cost effectiveness of gene expression profile testing in community practice. J Clin Oncol. 2018;36(6):554-562.

15. Huang JL, Kizy S, Marmor S, et al. Tumor grade and progesterone receptor status predict 21-gene recurrence score in early stage invasive breast carcinoma. Breast Cancer Res Treat. 2018. https://doi.org/10.1007/s10549-018-4955-z.

16. Gage MM, Rosman M, Mylander WC, et al. A validated model for identifying patients unlikely to benefit from the 21-gene recurrence score assay. Clin Breast Cancer. 2015;15(6):467-472.

17. Kim HS, Umbricht CB, Illei PB, et al. Optimizing the use of gene expression profiling in early-stage breast cancer. J Clin Oncol. 2016;34(36):4390-4397. 